\title{
MS03-05 | High-Throughput Stability Screening of Integral Membrane Proteins
}

Mlynek, Georg (University of Vienna MPL, Wien, AUT); Nagy, Michael (University of Vienna MPL, Wien, AUT); Kostan, Julius (Department für Strukturbiologie und Computational Biology, AUT); Kotov, Vadim (University Medical Center HamburgEppendorf (UKE), Hamburg, AUT); Marlovits, Thomas C. (University Medical Center Hamburg-Eppendorf (UKE), Hamburg, GER); Djinovic-Carugo, Kristina (University of Vienna MPL, Vienna, AUT)

Structural analysis has changed the way we look at our world. The foremost technique used in structural analysis is X-ray crystallography. When applied on biological macromolecules the major bottleneck of this method is the production of well diffracting crystals [1]. As structural biology projects are becoming increasingly challenging, the failure in the crystallization step is even more significant [2]. Therefore, there is a strong requirement for improvement of methods to enable the growth of diffraction quality crystals.

Recently a number of studies tried to capture key factors that affect protein crystallization by either tapping the large amount of data available on macromolecular structures, or by experimental findings $[3,4]$.

Besides the osmotic second virial coefficient $\left(B_{22}\right)$ [5] and monodispersity of protein solutions [6], protein thermal stability was shown to be a valuable criterion for assessment of crystallization propensity $[7,8]$. The rationale behind is that higher stability of the protein leads to higher amounts of chemically and conformationally pure samples throughout the purification process [8] and therefore to higher crystallization propensity as shown by systematic analysis of proteins by the high-throughput crystallization facility at EMBL-Grenoble [7].

The focus of this contribution is on the use of (nano)DSF for sample optimization and design of customized crystallization approaches. Additionally, emerging new concepts which employ analysis of thermodynamic data will be discussed.

[1] Derewenda and Vekilov, 2006

[2] Vincentelli and Romier, 2013

[3] Fusco et al., 2014

[4] Price et al., 2009

[5] Wilson and Delucas, 2014

[6] Proteau et al., 2010

[7] Dupeux et al., 2011

[8] Senisterra and Finerty, 2009 